\title{
Postoperative Alterations in Taste and Smell
}

\author{
Kelly Galina Elterman ${ }^{1}$; Seshagiri Rao Mallampati ${ }^{1}$; Alan David Kaye ${ }^{2}$; Richard Dennis \\ Urman ${ }^{1, *}$ \\ ${ }^{1}$ Department of Anesthesiology, Perioperative and Pain Medicine, Brigham and Women's Hospital, Boston, USA \\ ${ }^{2}$ Department of Anesthesiology, School of Medicine, Louisiana State University, New Orleans, USA \\ ${ }^{*}$ Corresponding author: Richard Dennis Urman, Department of Anesthesiology, Perioperative and Pain Medicine, Brigham and Women's Hospital, Boston, MA, USA. Tel: +1-16177328222, \\ Fax:+1-16172772192, E-mail: urmanr@gmail.com
}

Received: February 24, 2014; Accepted: March 16, 2014

\begin{abstract}
Context: Alterations in taste and smell, including but not limited to anosmia, ageusia, hypogeusia, and dysgeusia, have been described in association with various medications, including anesthetic agents. Frequently, these symptoms occur 1-2 weeks after medication administration and last several months. While such a phenomenon is a rare occurrence, it nonetheless can significantly impact patients' satisfaction and quality of life.

Evidence Acquisition: The methodology consisted of a thorough literature search using the MEDLINE and Cochrane databases utilizing keywords such as anosmia, ageusia, olfactory disorders, postoperative, and anesthesia.

Results: Our results yielded several previously published case report, and were not limited to a specific type of anesthesia. Based on available literature, we review the physiology of taste and smell as well as the medications associated with loss of these senses. We describe perioperative agents that could lead to postoperative complications associated with anosmia and and ageusia.

Conclusions: Based on available literature recommendations for anesthesiologists caring for patients at risk for this occurrence are presented in this review. The symptoms are usually temporary as in the majority of the patients the sensory receptor cells are able to regenerate themselves after injury. Anesthesia providers need to aware of this phenomenon to be able to reassure patients and possibly avoid anesthetic techniques associated with anosmia and ageusia.
\end{abstract}

Keywords: Olfaction Disorders; Ageusia; Anosmia; Postoperative Complications

\section{Context}

Anosmia, the loss of smell, and ageusia, the loss of taste, are rare side effects that can occur as a result of administration of practically all classes of medications $(1,2)$. The overall incidence of this rare complication has been estimated to be $0.05 \%$ and is frequently associated with cardiovascular medications (3). However, anesthetic drugs have also been implicated in cases of anosmia and/or ageusia. In this regard, the incidence of anosmia or ageusia after an anesthetic has been estimated to be approximately $1.8 \%$ (4). Regardless of cause, these symptoms, albeit usually lasting only 1-2 weeks, can greatly impact one's quality of life. Patients with anosmia and ageusia have been known to increase long-term intake of sugar and salt, resulting in worsening of chronic medical conditions such as diabetes mellitus, renal disease, and hypertensive disorders (5). The purpose of this review, based on the available peer-reviewed literature, is to summarize the physiology of taste, smell, gustation and olfaction, and to perform a literature review for prior reports of postoperative alterations in taste and smell. Finally, we analyze the available clinical evidence to make recommendations to anesthesia practitioners.

\section{Evidence Acquisition}

\subsection{Review of the Literature}

To date, there have been several case reports describing the phenomenon of postoperative anosmia and/ or ageusia, but no comprehensive review on the topic. As part of the methodology of this review, the MEDLINE/PubMed database was searched for papers using the terms, "anosmia," "ageusia," "olfactory disorders," "postoperative,"and "anesthesia," both alone and in various combinations to maximize results. Results were limited to papers written in English regarding humans. Results yielded several case reports, and were not limited to a specific type of anesthesia. The Cochrane Database was also queried using these search terms, but did not yield any significant results.

\subsection{Physiology of Taste and Smell}

The senses of taste and smell, also known as gustation and olfaction, respectively, are collectively known as "chemical senses" because of their ability to transmit chemical stimuli as a neural signal (6). These senses, al-

Copyright (c) 2014, Iranian Society of Regional Anesthesia and Pain Medicine (ISRAPM); Published by Kowsar. This is an open-access article distributed under the terms of the Creative Commons Attribution-NonCommercial 4.0 International License (http://creativecommons.org/licenses/by-nc/4.0/) which permits copy and redistribute the material just in noncommercial usages, provided the original work is properly cited. 
though they may seem trivial, are crucial to one's perception of the environment and quality of life.

\subsection{Gustation}

Taste occurs when a chemical substance stimulates a gustatory receptor cell within a taste bud. Taste buds are located primarily on the tongue, but are also found on the epithelial surface of the oropharynx and larynx. The taste bud contains spindle-shaped cells of three varieties: 1) the taste receptor cell, which is responsible for interacting with chemical stimuli; 2) the edge cell, which forms the lateral boundary of the taste bud; and 3) the basal cell, which forms the base of the taste bud and can proliferate to replace taste receptor cells. Typically, taste receptor cells are replaced approximately every 2 weeks (7). The taste bud is concentrically arranged around a pore that opens through the epithelium into the oropharynx. Apical microvilli allow gustatory stimuli to interact with the taste receptor cells. Afferent nerve fibers from cranial nerves VII, IX, and X penetrate the basal surface of the taste bud to innervate the taste receptor cell. A single cell may be innervated by multiple afferent nerve fibers, and a single fiber may synapse with a multitude of cells. Although they are modified epithelial cells and not quite neurons in vertebrates, taste receptor cells are similar to neurons (6). Taste buds on the tongue are found in 3 different types of papillae, which together house nearly 5000 taste buds. Collectively, taste buds are able to transmit information regarding 4 principal taste qualities: salt, sweet, bitter, and acid. Umami, a fifth taste quality, has also been described, and has been found to be associated with glutamate and inosinic acid. All taste buds are able to respond to all taste qualities. Importantly, saliva is necessary for transmission of taste stimuli as it helps transport solubilized taste molecules to the taste buds, and then wash those molecules away once stimulation of taste cell receptors has occurred (6). Stimulation of a taste receptor cell by an ingested taste molecule results in either depolarization or hyperpolarization of the taste receptor cell. With depolarization, there is a rise in intracellular calcium, leading to a release of a yet unknown neurotransmitter from the taste receptor cell to the afferent nerve fiber. Transmission of sweet, bitter and Umami involves G-protein-coupled second messengers. After stimulation of multiple receptors by a variety of taste particles, processing of all signals occurs. This allows for the simultaneous appreciation of different tastes. Additionally, processing can be inhibitory in that one region of the tongue can inhibit stimulation from adjacent regions. Adaptation also occurs, such that prolonged stimulation of one taste quality will decrease subsequent stimulation of the same taste $(6,7)$.

\subsection{Olfaction}

The sense of smell is similar to that of taste in that it also responds to chemical stimulation. Odorant molecules presented to the nasopharynx bind to olfactory receptors, which are G-protein coupled receptors, found in the cilia of olfactory receptor neurons in the neuroepithelium (Figure 1). The method of presentation of odorant molecules is important, in that velocity, air volume, and airflow direction can all affect smell perception (6). Further, retronasal olfaction, which is the passage of odorant molecules from the oropharyngeal cavity into the nose, allows for improved gustation.

Presentation of an olfactory molecule to the olfactory receptor results in depolarization of the cell, leading to propagation of an action potential to the olfactory bulb. There are approximately 1000 olfactory receptors; however, each can respond to multiple stimuli and a single odor can activate several types of receptors, resulting in the possibility for billions of combinations. As with taste, adaptation occurs such that prolonged exposure to a smell results in decreased stimulation. Importantly, unlike other neurons, the neurons of the olfactory and gustatory systems are able to replace themselves over the course of one's life.

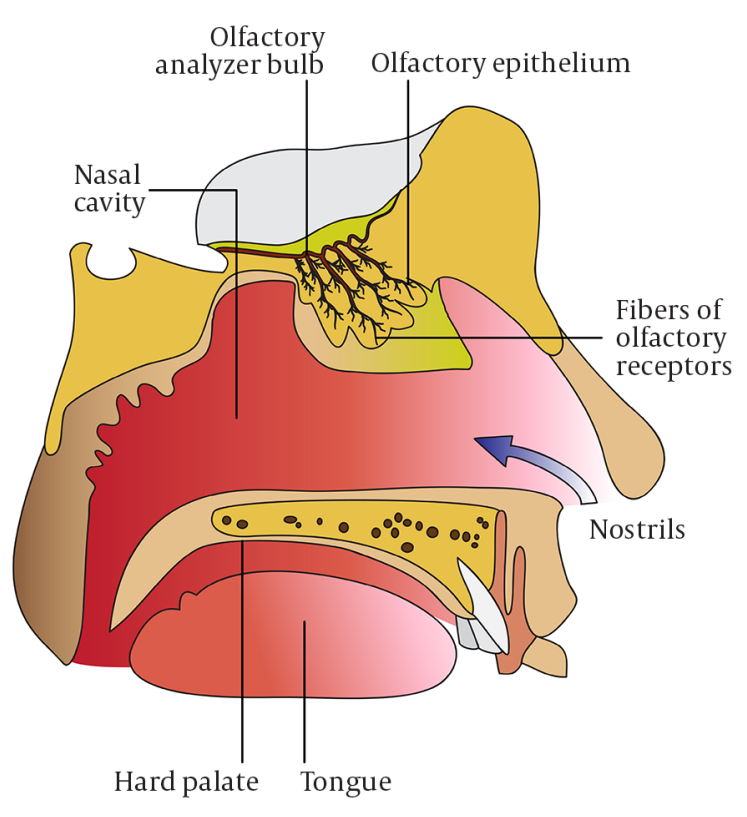

Figure 1. Illustration of Olfactory Nerves

\section{Results}

\subsection{Postoperative Alteration of Smell}

Postoperative anosmia has been described in several different case reports (Table 1). The only common offenders appear to be the use of general anesthetics, topical application of local anesthetics, and the undergoing of a surgical procedure, although no single procedure has been repeatedly linked to this phenomenon. Anosmia occurring after regional anesthesia has not been described. 
Elterman KG et al.

\begin{tabular}{|c|c|}
\hline Case Report/Study & Relevant Findings \\
\hline Green et al. 2008 (5) & Anosmia and ageusia after a stroke \\
\hline Konstantinidis et al. 2009 (8) & Four months of anosmia after GA in a 60-year-old man, with return of function after retraining. \\
\hline Dhanani and Jiang 2012 (9) & Six months of anosmia in a 57-year-old woman after GA for laparoscopic cholecystectomy. \\
\hline Carr et al. $2004(10)$ & Nasal irritation after intranasal ketamine. \\
\hline Mayell and Natusch 2009 (11) & Permanent anosmia after intranasal ketamine for pain management in a chronic pain patient. \\
\hline Salvinelli et al.2005 (12) & $\begin{array}{l}\text { Permanent anosmia after intranasal administration of } 4 \% \text { lidocaine, presumed due to contact of } \\
\text { the local anesthetic to the olfactory cleft. }\end{array}$ \\
\hline Welge-Lüssen et al. 2004 (13) & $\begin{array}{l}\text { Intranasal tetracaine decreased subjective assessment of smell, but olfactory event-related } \\
\text { potentials and chemosensory event-related potentials were unchanged. }\end{array}$ \\
\hline Cooper $1998(14)$ & Resolution of 20-year-long anosmia after GA. \\
\hline Cassidy and McCoy 2000 (15) & Resolution of 15-year-long anosmia after ESI. \\
\hline Cardoso et al. $2007(16)$ & Ageusia lasting 3 weeks after LMA use \\
\hline Koyama et al. $2006(17)$ & Ageusia lasting 6 months after LMA use \\
\hline Hotta et al. $2002(18)$ & $\begin{array}{c}\text { Ipsilateral loss of taste and atrophy of fungiform papillae after local anesthetic injection near } \\
\text { inferior alveolar nerve. }\end{array}$ \\
\hline
\end{tabular}

In a patient in which 4-month long anosmia occurred in a 60 year-old man who underwent a general anesthetic with propofol, fentanyl, and sevoflurane, postoperative brain imaging demonstrated no evidence of lesion or decreased function. The patient's sense of smell returned after retraining (8). In a similar case, a 57-year-old woman developed anosmia for 6 months following a routine laparoscopic cholecystectomy under general anesthesia (9). Intranasal administration of medications, specifically ketamine, has resulted in both transient and permanent complications. Carr et al. reported the occurrence of transient nasal irritation after intranasal ketamine use, (10) while Mayell et al. described a patient who developed permanent anosmia after the initiation of intranasal ketamine for analgesia in a chronic pain patient (11). Patients have also developed anosmia after the topical use of local anesthetics. Salvinelli et al. reported on a 62 year-old man who developed permanent anosmia after use of intranasal $4 \%$ lidocaine. It was hypothesized that anosmia may have been related to contact of local anesthetic with the olfactory cleft (Figure 1). This interaction may have resulted from positioning (12). Interestingly, Welge-Lüssen et al. showed that while intranasal tetracaine decreased subjective assessment of smell, analysis of olfactory eventrelated potentials and chematosensory event-related potentials revealed that it was largely unchanged (13). Although each of these cases is slightly different, the common thread seems to be destruction of olfactory-receptor cells, leading to alteration or complete loss of smell. Given that these cells have the ability to regenerate, it is not surprising that in most cases the sense returns over a period of time. The rare cases of permanent anosmia may be related to degree of initial injury to olfactory receptor cells or the individual patient's inability to regenerate those cells. Perhaps what is most interesting, however, is that reversal of previously longstanding anosmia after anesthesia has been reported. Cooper reported a patient with a 20-year history of anosmia whose sense of smell spontaneously returned after general anesthesia with propofol, morphine, isoflurane, vecuronium, and nitrous oxide (14). Similarly, Cassidy and McCoy reported a woman with a 15 year history of anosmia whose symptoms resolved after epidural steroid injection (15). Both were at a loss for explanation of the mechanism.

\subsection{Postoperative Alterations of Taste}

Postoperative ageusia or dysgeusia has also been described (Table 1), albeit with less frequency than anosmia and seemingly a different mechanism. Cardoso et al. reported the case of ageusia lasting 3 weeks after use of a laryngeal mask airway (LMA) inflated to $30 \mathrm{cc}$ for breast surgery (16). The authors hypothesized that the etiology was compression of the lingual nerve. Similarly, Koyama and colleagues described a patient who suffered ageusia for 6 months after LMA use (17). Local anesthetic injected near the inferior alveolar nerve for dental procedures has resulted in ipsilateral loss of taste and atrophy of fungiform papillae (18). In both cases, symptoms were noted 3 months after a dental procedure, with complete resolution by 13 months.

\section{Conclusions}

While post-operative anosmia or ageusia may be a relatively rare occurrence, it can alter a patient's quality of life and lead to both social and medical consequences. For example, Green et al. described the case of a patient who suffered a stroke, which resulted in anosmia and ageusia (5). As this interfered with quality of life, the patient compensated by increasing salting and sweetening of foods, which led to worsening of underlying chronic conditions. Patients suffering from anosmia or ageusia 
postoperatively, rather than as a result of a cerebrovascular accident, may also adjust their diets in a similar way, and worsen underlying conditions such as diabetes or hypertension. For this reason, anesthesiologists should consider this possibility when caring for patients, particularly those at risk for or with history of olfactory or gustatory abnormalities. Potential methods of avoiding or minimizing the risk of anosmia include avoidance of intranasal ketamine, limitation of the duration of exposure of the olfactory cleft to lidocaine at concentrations equal to or greater than $4 \%$, or use of lidocaine at a concentration of less than $4 \%$. Similarly, avoidance of LMA over-inflation, using volumes less than $30 \mathrm{cc}$, and limitation of LMA use to short surgical procedures, may all potentially help reduce the risk of ageusia. While anesthesiologists can certainly attempt to take some measures to minimize the occurrence of this phenomenon in patients deemed to be at risk, it must be emphasized that as no single etiology exists, it may be impossible to predict or reliably prevent the occurrence of anosmia or ageusia. For this reason, it is important to be able to recognize this condition and understand that its pathophysiology is taste receptor cell or olfactory receptor cell destruction. It is equally important to realize that these cells regenerate in most cases and, thus the course of these symptoms is typically transient. Armed with this knowledge, anesthesiologists faced with such a postoperative dilemma can provide reassurance and counsel patients appropriately.

Taste and smell are seemingly trivial senses, the loss of which however can significantly affect one's quality of life, particularly if the loss results in alteration of diet. Although rare, loss of these senses has been reported to occur after administration of various anesthetic agents. Fortunately, the symptoms are usually temporary as in the majority of patients the sensory receptor cells are able to regenerate themselves after injury. Anesthesiologists ought to be aware of this phenomenon so that they may be able to reassure patients, and avoid anesthetic techniques associated with anosmia and/or ageusia when caring for patients who have previously experienced alterations in taste or smell, postoperatively or otherwise.

\section{Authors' Contributions}

Richard Urman created the idea for this manuscript. Kelly Elterman performed the literature review, and wrote as well as edited the manuscript. S. Rao Mallampati and Alan D. Kaye also assisted with the writing and editing of the manuscript. All authors contributed to the literature review and preparation of this manuscript.

\section{References}

1. Henkin RI. Drug-induced taste and smell disorders. Incidence, mechanisms and management related primarily to treatment of sensory receptor dysfunction. Drug Saf. 1994;11(5):318-77.

2. Doty RL, Bromley SM. Effects of drugs on olfaction and taste. Otolaryngol Clin North Am. 2004;37(6):1229-54

3. Nores JM, Biacabe B, Bonfils P. [Olfactory disorders due to medications: analysis and review of the literature]. Rev Med Interne. 2000;21(11):972-7.

4. Kostopanagiotou G, Kalimeris K, Kesidis K, Matsota P, Dima C, Economou M, et al. Sevoflurane impairs post-operative olfactory memory but preserves olfactory function. EurJ Anaesthesiol. 2011;28(1):63-8.

5. Green TL, McGregor LD, King KM. Smell and taste dysfunction following minor stroke: a case report. Can J Neurosci Nurs. 2008;30(2):10-3.

6. Hadley K, Orlandi RR, Fong KJ. Basic anatomy and physiology of olfaction and taste. Otolaryngol Clin North Am. 2004;37(6):1115-26.

7. Yarmolinsky DA, Zuker CS, Ryba NJ. Common sense about taste: from mammals to insects. Cell. 2009;139(2):234-44.

8. Konstantinidis I, Tsakiropoulou E, Iakovou I, Douvantzi A, Metaxas S. Anosmia after general anaesthesia: a case report. Anaesthesia. 2009;64(12):1367-70.

9. Dhanani NM, Jiang Y. Anosmia and hypogeusia as a complication of general anesthesia. J Clin Anesth. 2012;24(3):231-3.

10. Carr DB, Goudas LC, Denman WT, Brookoff D, Staats PS, Brennen L, et al. Safety and efficacy of intranasal ketamine for the treatment of breakthrough pain in patients with chronic pain: a randomized, double-blind, placebo-controlled, crossover study. Pain. 2004;108(1-2):17-27.

11. Mayell A, Natusch D. Anosmia--a potential complication of intranasal ketamine. Anaesthesia. 2009;64(4):457-8.

12. Salvinelli F, Casale M, Hardy JF, D'Ascanio L, Agro F. Permanent anosmia after topical nasal anaesthesia with lidocaine $4 \% . \mathrm{Br} J$ Anaesth. 2005;95(6):838-9.

13. Welge-Lussen A, Wille C, Renner B, Kobal G. Anesthesia affects olfaction and chemosensory event-related potentials. Clin Neurophysiol. 2004;115(6):1384-91.

14. Cooper GM. An unexpected benefit of anaesthesia. Anaesthesia. 1998;53(8):830.

15. Cassidy B, McCoy DG. The sweet smell of success. Anaesthesia 2000;55(8):823.

16. Cardoso HE, Kraychete DC, Lima Filho JA, Garrido LS, Rocha AP. [Temporary lingual nerve dysfunction following the use of the laryngeal mask airway: report]. Rev Bras Anestesiol. 2007;57(4):410-3.

17. Koyama T, Ichizawa A, Fukami N, Arai K, Hirata S, Mishima S. [Taste loss following the use of the laryngeal mask airway]. Masui. 2006;55(4):445-6.

18. Hotta M, Endo S, Tomita H. Taste disturbance in two patients after dental anesthesia by inferior alveolar nerve block. Acta Otolaryngol Suppl. 2002(546):94-8. 